Correction

\title{
Correction: Brad et al. The Chemoautotrophically Based Movile Cave Groundwater Ecosystem, a Hotspot of Subterranean Biodiversity. Diversity 2021, 13, 128
}

\author{
Traian Brad 1,2,(D), Sanda Iepure 1,2,(1) and Serban M. Sarbu 3,4,*, (D) \\ 1 “Emil Racovită" Institute of Speleology, Str. Clinicilor nr. 5-7, 400006 Cluj-Napoca, Romania; \\ traian.brad@academia-cj.ro (T.B.); sanda.iepure@academia-cj.ro (S.I.) \\ 2 Institutul Român de Știință și Tehnologie, Str. Virgil Fulicea nr. 3, 400022 Cluj-Napoca, Romania \\ "Emil Racovită" Institute of Speleology, Str. Frumoasa nr. 31, 010986 București, Romania \\ 4 Department of Biological Sciences, California State University, Chico, CA 95929, USA \\ * Correspondence: serban.sarbu@yahoo.com
}

check for updates

Citation: Brad, T.; Iepure, S.; Sarbu, S.M. Correction: Brad et al. The Chemoautotrophically Based Movile Cave Groundwater Ecosystem, a Hotspot of Subterranean Biodiversity. Diversity 2021, 13, 128. Diversity 2021, 13, 461. https://doi.org/10.3390/ d13100461

Received: 21 May 2021

Accepted: 4 September 2021

Published: 24 September 2021

Publisher's Note: MDPI stays neutral with regard to jurisdictional claims in published maps and institutional affiliations.

Copyright: (c) 2021 by the authors. Licensee MDPI, Basel, Switzerland. This article is an open access article distributed under the terms and conditions of the Creative Commons Attribution (CC BY) license (https:// creativecommons.org/licenses/by/ $4.0 /)$.
The authors wish to make the following corrections to this paper [1]. The authors apologize for any inconvenience caused and state that the scientific conclusions are unaffected.

1. Replacing a sentence in Section 2.2 Terrestrial fauna, on page 5:

The geophilid centipedes Geophilus sp. and Clinopodes carynthiacus are also among the predators in this ecosystem.

With

The geophilid centipedes Geophilus sp. and Clinopodes carinthiacus are also among the predators in this ecosystem.

2. Replacing a sentence in Section 2.2 Terrestrial fauna, on page 6:

The three species jump continuously in all directions, and therefore they can easily become part of the menu of other cave inhabitants.

With

Two of the three species jump continuously in all directions; therefore, they can easily become part of the menu of other cave inhabitants.

3. Splitting of Table 2 (List of aquatic and terrestrial invertebrate species encountered and described in Movile Cave ecosystem) into troglobionts/stygobionts (Table 2) and troglophiles/stygophiles (Table 3), as following:

4. Replacing a sentence in Section 2.2 Terrestrial fauna, on page 9:

Caucasonethes vandeli pygmaeus is an extremely small isopod, about $1 \mathrm{~mm}$ long, it is translucid, and moves very fast.

With

Caucasonethes vandeli pygmaeus is an extremely small isopod, less than $2 \mathrm{~mm}$ long, it is translucid, and moves very fast.

5. Replacing a sentence in the Section: Funding on page 10:

S. Iepure and S. Sarbu were supported by grant PN-III-P4-ID-PCE-2020-2843 (EVODEVO-CAVE).

Should be replaced with

S. Iepure and S. Sarbu were supported by grants of Ministry of Research and Innovation (UEFISCDI) projects number PN-III-P4-ID-PCE-2020-2843 (EVO-DEVO-CAVE) and PN-III-P4-ID-PCCF-2016-0016 (DARKFOOD). 
Table 2. List of troglobionts and stygobionts from Movile Cave.

\begin{tabular}{|c|c|c|c|c|}
\hline & Aquatic/Terrestrial & Species & Taxonomic Affiliation & References \\
\hline 1 & Aquatic & Dendrocoelum obstinatum *; Stocchino et al., 2017 & Platyhelminthes, Dendrocoelidae & [30] \\
\hline 2 & Aquatic & Panagrolaimus cf. thienemani ${ }^{*}$ & Nematoda, Panagrolaimidae & [34] \\
\hline 3 & Aquatic & Chronogaster troglodytes *; Poinar and Sarbu, 1994 & Nematoda, Chronogasteridae & [35] \\
\hline 4 & Aquatic & Haemopis caeca ${ }^{*}$; ; Manoleli et al., 1998 & Annelida, Hirudinea, Haemopidae & [36] \\
\hline 5 & Aquatic & Helodrilus sp. nov. * & Annelida, Clitellata, Lumbricidae & Martin, P., pers. comm. \\
\hline 6 & Aquatic & Heleobia dobrogica *; Grossu and Negrea, 1989 & Gastropoda, Moitessieriidae & [29] \\
\hline 7 & Aquatic & Pseudocandona sp. nov. ${ }^{*}$ & Crustacea, Ostracoda, Cyprididae & Danielopol, D., pers. comm. \\
\hline 8 & Aquatic & Eucyclops graeteri scythicus *; Plesa, 1989 & Crustacea, Copepoda, Cyclopidae & [37] \\
\hline 9 & Aquatic & Parapseudoleptomesochra italica; Pesce and Petkovski, 1980 & Crustacea, Copepoda, Harpacticoida & Rouch, pers. comm. \\
\hline 10 & Aquatic & Niphargus racovitzai *; Dancau, 1970 & Crustacea, Amphipoda, Niphargidae & [38] \\
\hline 11 & Aquatic & Niphargus dancaui *,\#; Brad et al., 2015 & Crustacea, Amphipoda, Niphargidae & [39] \\
\hline 12 & Aquatic & $\begin{array}{c}\text { Asellus aquaticus infernus *,\#; Turk-Prevorčnik and } \\
\text { Blejec, } 1998\end{array}$ & Crustacea, Isopoda, Asellidae & [40] \\
\hline 13 & Terrestrial & Caucasonethes vandeli pygmaeus *; Giurginca, 2020 & Crustacea, Isopoda, Trichoniscidae & [41] \\
\hline 14 & Terrestrial & Haplophthalmus movilae *; Gruia and Giurginca, 1998 & Crustacea, Isopoda, Trichoniscidae & [42] \\
\hline 15 & Terrestrial & Trachelipus troglobius *; Tabacaru and Boghean, 1989 & Crustacea, Isopoda, Trachelipodidae & [43] \\
\hline 16 & Terrestrial & Armadillidium tabacarui *; Gruia et al., 1994 & Crustacea, Isopoda, Armadillidiidae & [44] \\
\hline 17 & Terrestrial & Chthonius monicae *; Boghean, 1989 & Arachnida, Pseudoscorpiones, Chthoniidae & [45] \\
\hline 18 & Terrestrial & Chthonius borissketi ${ }^{*}$; Curčić et al., 2014 & Arachnida, Pseudoscorpiones, Chthoniidae & [46] \\
\hline 19 & Terrestrial & Roncus dragobete *; Curčić et al., 1993 & Arachnida, Pseudoscorpiones, Neobisiidae & [47] \\
\hline 20 & Terrestrial & Roncus ciobanmos *; Curčić et al., 1993 & Arachnida, Pseudoscorpiones, Neobisiidae & [47] \\
\hline 21 & Terrestrial & Palliduphantes constantinescui *; Georgescu, 1989 & Arachnida, Araneae, Linyphiidae & [48] \\
\hline 22 & Terrestrial & Agraecina cristiani ${ }^{*}, \#$; Georgescu, 1989 & Arachnida, Araneae, Liocranidae & [48] \\
\hline 23 & Terrestrial & Kryptonesticus georgescuae *; Nae, Sarbu, and Weiss, 2018 & Arachnida, Araneae, Nesticidae & [49] \\
\hline 24 & Terrestrial & Hahnia caeca*; Georgescu and Sarbu, 1992 & Arachnida, Araneae, Hahniidae & [50] \\
\hline 25 & Terrestrial & Labidostomma motasi *; Iavorschi, 1992 & Arachnida, Acarina, Labidostommatidae & [51] \\
\hline 26 & Terrestrial & Geophilus sp. nov. * & Chilopoda, Geophilidae & Baba, St., pers. comm. \\
\hline 27 & Terrestrial & 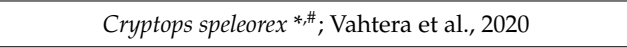 & Chilopoda, Cryptopidae & [31] \\
\hline 28 & Terrestrial & Archiboreoiulus serbansarbui ${ }^{*, \#}$; Giurginca et al., 2020 & Diplopoda, Julida, Julidae & [52] \\
\hline 29 & Terrestrial & Onychiurus movilae *; Gruia, 1989 & Collembola, Onychiuridae & [53] \\
\hline 30 & Terrestrial & Oncopodura vioreli *; Gruia, 1989 & Collembola, Oncopoduridae & [53] \\
\hline 31 & Terrestrial & Plusiocampa isterina *; Condé, 1993 & Diplura, Campodeidae & [54] \\
\hline 32 & Terrestrial & Plusiocampa euxina *; Condé, 1996 & Diplura, Campodeidae & [55] \\
\hline 33 & Terrestrial & Medon dobrogicus *; Decu and Georgescu, 1994 & Coleoptera, Staphylinidae & [56] \\
\hline 34 & Terrestrial & Tychobythinus sulphydricus *; Poggi and Sarbu, 2013 & Coleoptera, Staphylinidae & [57] \\
\hline 35 & Terrestrial & Decumarellus sarbui *; Poggi, 1994 & Coleoptera, Staphylinidae & [58] \\
\hline 36 & Terrestrial & Bryaxis dolosus *; Poggi and Sarbu, 2013 & Coleoptera, Staphylinidae & [57] \\
\hline 37 & Terrestrial & Clivina subterranea *; Decu et al., 1994 & Coleoptera, Clivinidae & [59] \\
\hline 38 & Aquatic & Nepa anophthalma *; Dedu et al., 1994 & Hemiptera, Nepidae & [60] \\
\hline
\end{tabular}

*-species endemic to Movile Cave; ${ }^{\#}$ - species found in nearby springs and wells. 
Table 3. List of troglophiles and stygophiles from Movile Cave.

\begin{tabular}{|c|c|c|c|c|}
\hline & Aquatic/Terrestrial & Species & Taxonomic Affiliation & References \\
\hline 1 & Aquatic & Udonchus tenuicaudatus; Cobb, 1913 & Nematoda, Rhabdolaimidae & [34] \\
\hline 2 & Aquatic & Poikilolaimus sp. & Nematoda, Rhabditidae & {$[34]$} \\
\hline 3 & Aquatic & Monhystrella sp. & Nematoda, Monhysteridae & [34] \\
\hline 4 & Aquatic & Habrotrocha rosa; Donner, 1949 & Rotatoria, Habrotrochidae & Ricci, C., pers. comm. \\
\hline 5 & Aquatic & Habrotrocha bidens; Gosse, 1851 & Rotatoria, Habrotrochidae & Ricci, C., pers. comm. \\
\hline 6 & Aquatic & Aelosoma hyalinum; Bunke, 1967 & Annelida, Aeolosomatidae & Dumnicka, E., pers. comm. \\
\hline 7 & Aquatic & Aelosoma italica; Bunke, 1967 & Annelida, Aeolosomatidae & Dumnicka, E., pers. comm. \\
\hline 8 & Aquatic & Tropocyclops prasinus; Fischer, 1860 & Crustacea, Copepoda, Cyclopidae & [37] \\
\hline 9 & Terrestrial & Carniella brignolii; Thaler and Steinberger, 1988 & Arachnida, Araneae, Theridiiae & [48] \\
\hline 10 & Terrestrial & Dysdera hungarica; Kulczynski, 1897 & Arachnida, Araneae, Dysderidae & Weiss, L., pers. comm. \\
\hline 11 & Terrestrial & Clinopodes carinthiacus; Latzel, 1880 & Chilopoda, Geophilidae & Zapparoli, M., pers. comm. \\
\hline 12 & Terrestrial & Strongylosoma jaqueti; Verhoeff, 1898 & Diplopoda, Paradoxosomatidae & Tajovsky K., pers comm. \\
\hline 13 & Terrestrial & Pygmarrhopalites pygmaeus; Wankel, 1860 & Collembola, Arrhopalitidae & [55] \\
\hline
\end{tabular}

\section{Reference}

1. Brad, T.; Iepure, S.; Sarbu, S.M. The Chemoautotrophically Based Movile Cave Groundwater Ecosystem, a Hotspot of Subterranean Biodiversity. Diversity 2021, 13, 128. [CrossRef] 